\title{
Clinical Usefulness of Magnetic Resonance Imaging in Four Children With Scleroderma
}

\author{
Kazushi AGATA, ${ }^{1}$ Hisashi KAWASHIMA,${ }^{1}$ Atsushi KUMADA, ${ }^{1}$ Yasuyo KASHIWAGI, ${ }^{1}$ Hiroko HARA ${ }^{2}$ \\ ${ }^{1}$ Department of Pediatrics, Tokyo Medical University, Tokyo, Japan \\ ${ }^{2}$ Department of Radiology, Kawaguchi Municipal Medical Center, Saitama, Japan
}

\begin{abstract}
Children with localized scleroderma may have more serious sequelae than adults with the disease. In this case report, we analyzed four girls with localized scleroderma (generalized morphea) to evaluate the clinical usefulness of magnetic resonance imaging (MRI) in facilitating disease control and investigating patient responsiveness to various treatments. Two of the children had flexion contracture, and two displayed a clear high-intensity area in the bone marrow under the sclerotic skin on MRI. Two girls had different bilateral circumferences of the legs or forearms. These abnormalities were detected without any obvious changes in blood chemistry. Our results demonstrated that MRI is useful for the evaluation of treatment effects for localized scleroderma in children.

Keywords: Localized scleroderma; magnetic resonance imaging; methotrexate; mycophenolate mofetil; osteomyelitis.
\end{abstract}

Localized scleroderma, also known as morphea, is an uncommon persistent condition (0.02 to 0.04 in 100,000 people) in which there are cutaneous lesions of thickened skin, as well as lesions occasionally in the underlying tissues, including bone. ${ }^{1}$ The most widely used classification divides morphea into five general subtypes as follows: plaque morphea, generalized morphea, linear scleroderma, bullous morphea, and deep morphea. ${ }^{2}$ Localized scleroderma is differentiated from systemic sclerosis by its supposed lack of internal organ involvement. Because most physicians are unaware of the severity of scleroderma owing to the lack of pain, patients with localized scleroderma are rarely referred to a pediatric rheumatologist. Several methods of treatment have been tested over the past decade for this intractable disease. Children with this disease have more serious sequelae than adults, because as they grow, the disease affects their joints and bones near the affected skin..$^{3-5}$ In this article, we report four children with localized scleroderma (generalized morphea), who were treated with methotrexate (MTX), steroids, or both, to evaluate the clinical usefulness of magnetic resonance imaging (MRI) in facilitating disease control and investigating patient responsiveness to various treatments.

\section{CASE REPORT}

Case 1- A 12-year-old girl applied with skin sclerosis. Her past medical and family histories were unremarkable. She first noticed skin sclerosis of her right leg in August 2005. The sclerosis extended rapidly from the dorsum of the foot to the hip, accompanied with decoloration. In March 2006, she was referred to our hospital and a skin biopsy was performed. She demonstrated a decreased range of motion in her right toe. Following a diagnosis of localized scleroderma,

Received: May 03, 2017 Accepted: August 31, 2017 Published online: November 02, 2017

Correspondence: Kazushi Agata, MD. Department of Pediatrics, Tokyo Medical University, 160-0023 Tokyo, Japan.

Tel: +81333426111 e-mail: whatwouldjesusdo721@yahoo.co.jp

๑2018 Turkish League Against Rheumatism. All rights reserved. 
she was treated with a combination of MTX and steroids. However, there was no improvement. She was admitted to our hospital for psoralen and ultraviolet A (PUVA) treatment in July 2006 (11 months since patient realized symptoms). This improved her skin abnormality and she was subsequently treated in the outpatient clinic. At the time of her original admission, she had sclerotic lesions on the lower abdomen, hips, lower extremities, and anterior leg, with areas of irregular pigmentation and depigmentation. Because of these symptoms, she was unable to sit properly. The metatarsophalangeal joints of the second to fourth toes and the right knee joint showed mild flexion contracture. The circumferences of the lower extremities differed bilaterally by $3 \mathrm{~cm}$ (modified localized scleroderma skin index [mLoSSI]: 24, localized scleroderma skin damage index [LoSDI]: 22). After 10 days of
PUVA treatments, the condition of her skin and the subjective symptom of tightness improved and she was able to sit properly. After her discharge, she was treated with MTX and steroids according to her blood test results, including soluble interleukin (IL)-2 receptor and antinuclear antibody levels. There was no worsening of her condition according to the blood test results.

The initial MRI of her lower leg (Figure 1a-d) taken in March 2012 showed generalized atrophy of the right lower leg and localized atrophy of the subcutaneous fat abutting the tibia. In the diaphysis of the right tibia, a high intensity area was visualized by $\mathrm{T}_{2}$-weighted short-tau inversion recovery (STIR) (Figure 1a), which showed hypointensity on $\mathrm{T}_{1}$-weighted imaging ( $\mathrm{T}_{1} \mathrm{WI}$ ) (Figure 1b), and was well enhanced on fatsaturated postgadolinium $\mathrm{T}_{1} \mathrm{WI}$ (Figure $1 \mathrm{c}$ and $\mathrm{d}$ ).
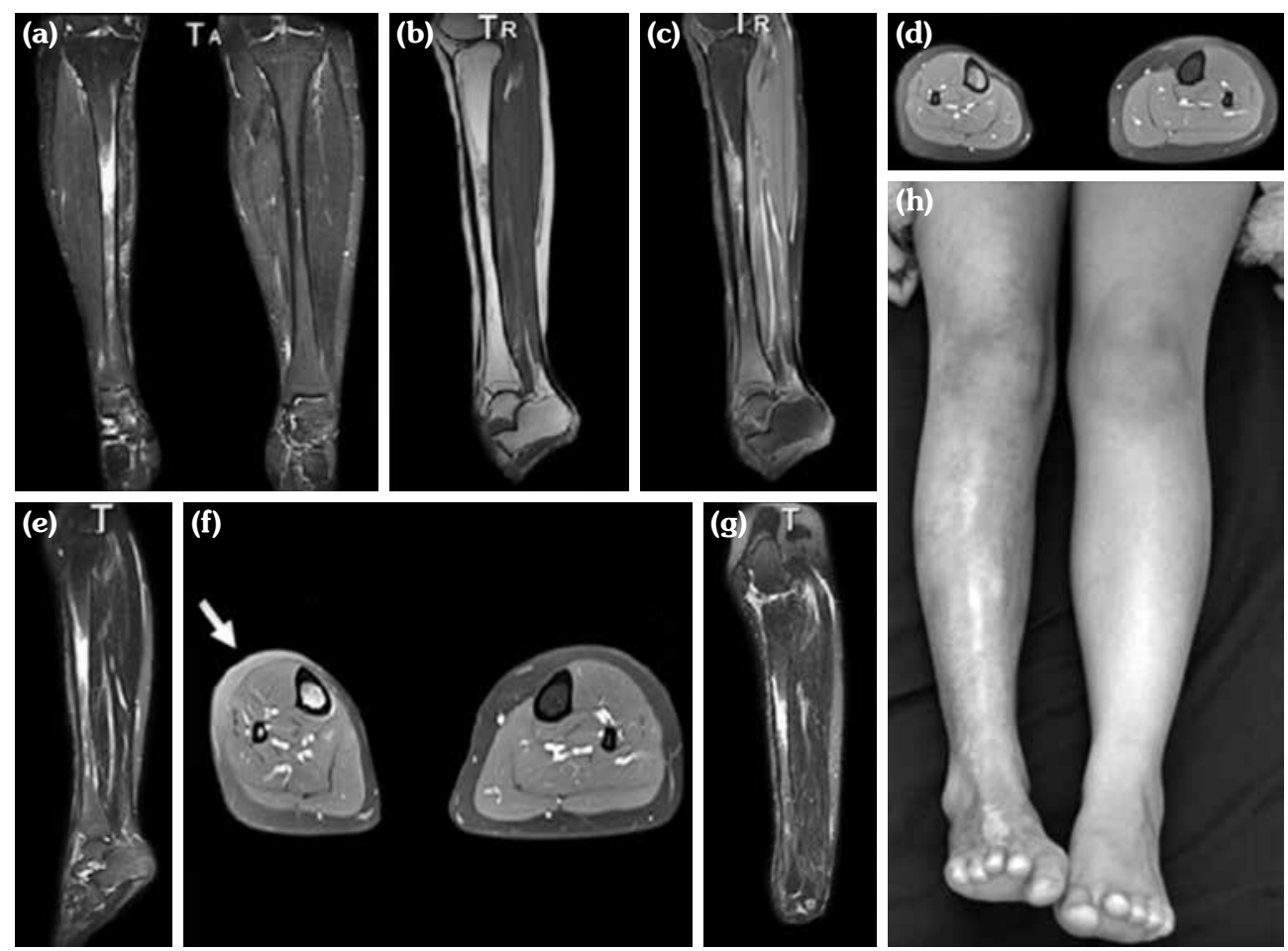

Figure 1. (a-d) Magnetic resonance imaging of case 1 (12-year-old girl). In initial magnetic resonance imaging, (a) a high intensity area was displayed on diaphysis of right tibia on coronal short-tau inversion recovery images. (b) Same lesion was displayed as low signal intensity on sagittal $\mathrm{T}_{1}$-weighted imaging, and well enhanced on fat-saturated postgadolinium (c) sagittal and (d) axial $\mathrm{T}_{1}$-weighted imaging. (e, f) In second magnetic resonance imaging performed three weeks later, (e) bone marrow lesion had scattered distally in tibia on sagittal short-tau inversion recovery. (f, arrow) Enhancement of subcutaneous soft tissue in anterior part of right lower leg was newly displayed on fat-saturated postgadolinium axial $\mathrm{T}_{1}$-weighted imaging. (g) Bone marrow lesion of tibia was reduced on short-tau inversion recovery in August. In $\mathbf{a}, \mathbf{d}, \mathbf{f}$, and $\mathbf{h}$, right lower leg was observed to be compared with left. 
The second MRI, performed three weeks later (Figure $1 e$ and $\mathrm{f}$ ), showed that the bone marrow lesion was further scattered in the tibia. Enhancement of the subcutaneous soft tissue in the anterior part of the right lower leg was newly displayed on fat-saturated postgadolinium axial $\mathrm{T}_{1} \mathrm{WI}$. Therefore, we increased the dose of MTX. As findings from the MRI performed in May 2012 (not shown) had not improved despite increasing the dose of MTX, MTX was increased further. Between August and September, the patient's skin sclerosis improved, and a reduction in the size of the bone marrow lesion was confirmed on STIR (Figure 1g). A written informed consent was obtained from the patient.

Case 2- A 13-year-old girl complained of a pigmented patch on the back of her left hand as well as other places. Her birth and past medical history were unremarkable. The area of sclerotic skin on the dorsum of her left hand, which first appeared at three years of age, grew larger and a dermatologist was consulted when the patient was four years old. The range of motion of her left third finger was limited. The laboratory findings were positive for antinuclear antibodies at a dilution of 1:160 (speckled and homogenous) and a high
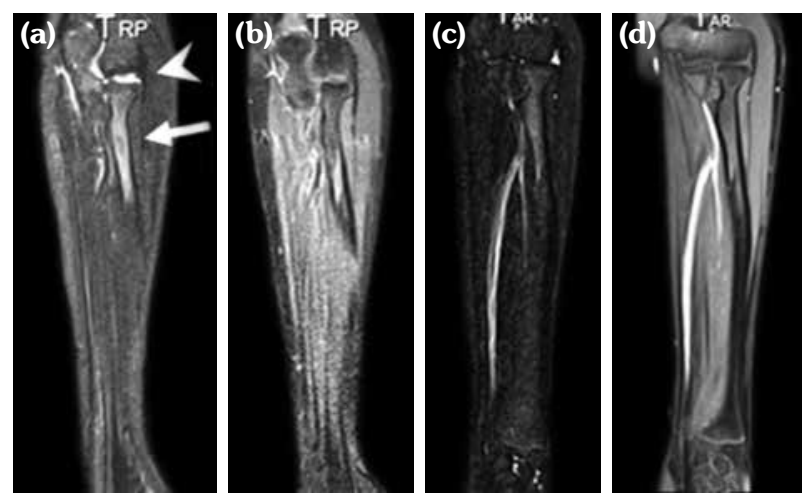

Figure 2. Magnetic resonance imaging of case 2 (13-year-old girl with plaque morphea). (a, b) In initial magnetic resonance imaging taken in March 2012, a high intensity area was displayed in metaphysis to diaphysis of left proximal radius on coronal short-tau inversion recovery (a, arrow) with faint contrast enhancement on fat-saturated postgadolinium coronal $\mathrm{T}_{1}$-weighted imaging $(\mathbf{b})$, suggesting bone marrow involvement. A small amount of joint effusion (a, arrowhead) was also displayed in elbow joint, and steroid treatment was started again. As no significant improvement was observed on magnetic resonance imaging performed four months later ((c), coronal short-tau inversion recovery; (d), fat-saturated coronal $\mathrm{T}_{1}$-weighted imaging) steroid treatment was continued. level of soluble IL-2 receptor $(852 \mathrm{U} / \mathrm{mL})$ with her blood test results. Her condition was diagnosed as plaque scleroderma and the patient was treated with MTX and daily doses of prednisolone (one year since patient realized symptoms). After starting treatment, the lesions became smaller and her sclerotic condition improved. Steroid treatment was hence tapered and then stopped. Until the age of 13 years, there were no changes in blood test values and no apparent symptoms; her condition was managed only by weekly MTX. At 13 years of age, the sclerotic lesions on the upper extremities became worse. Well-circumscribed sclerotic skin lesions appeared on the dorsum of the left hand, proximal portion of the left brachium, and cubital fossa, irregularly mixed with areas of hyperpigmentation and central hypopigmentation (mLoSSI: 15, LoSDI: 17). There was a lilac ring on the dorsum of her left hand, although there were no abnormalities noted by blood tests. The levels of soluble IL-2 receptor had decreased to normal levels.

The initial MRI (Figure 2a and b) in March 2012 displayed a high-intensity area in the metaphysis to diaphysis of the left proximal radius on STIR (Figure 2a, arrow) with faint contrast enhancement on fat-saturated $\mathrm{T}_{1} \mathrm{WI}$ (Figure 2b), suggesting bone marrow involvement. A small amount of joint effusion (Figure 2a, arrowhead) was also observed in the elbow joint, steroid treatment was started again. As no significant improvement was displayed on the MRI taken four months later in July 2012 (Figure 2c and d), steroid treatment was continued. A written informed consent was obtained from the patient and her parent.

Case 3- An 11-year-old girl complained of skin hardening, frequent occurrences of joint contracture of the fingers, elbow, wrist, and neck, as well as gait disturbance. Her past medical and family histories were unremarkable. She had noticed a hardening of her skin and movement difficulties in her hand joints from March 1993, at the age of nine years. In May 1993, a decreased range of motion in her right fourth and fifth fingers appeared, and she consulted her family doctor. Although ointment and massage treatment were provided, her symptoms were progressive and she was hospitalized in June 1993. Her condition was diagnosed as localized scleroderma by skin biopsy. 
An ointment for external application, nonsteroidal antiinflammatory drugs, and rehabilitation treatments were administered. However, because her skin and joint conditions became worse and she had a sudden loss of weight, she was admitted to our hospital in October 1995 at the age of 11 years. On admission, she showed neck joint contracture to the right, as well as contracture of the third and fourth right fingers, third left finger, and right elbow at fixed positions. A thickening and tightening of the skin, of 4 to $15 \mathrm{~cm}$ in diameter, was noted on her back, elbow, and extremities (mLoSSI: 43, LoSDI: 45). Laboratory findings on admission were normal with the exception of an erythrocyte sedimentation rate of $22 \mathrm{~mm} /$ hour, and positive antinuclear antibodies at a dilution of 1:40 (speckled/ nucleolar). Her blood test results demonstrated an immunoglobulin $\mathrm{G}$ level of $1,590 \mathrm{mg} / \mathrm{dL}$. After admission, MTX was intravenously administered at a dose of $30 \mathrm{mg}(1.2 \mathrm{mg} / \mathrm{kg} /$ week $)$ every week for six weeks (two years and seven months since patient realized symptoms), followed by a dose of $10 \mathrm{mg}(0.4 \mathrm{mg} / \mathrm{kg} /$ week $)$ orally once a week, up to the present. One week after her admission, the torticollis, sclerosis, and pigmentation of her skin improved. The range of motion of the neck, elbow, and knee joints also improved slightly, and her weight increased. However, the range of motion of her fingers and hands did not improve. Therefore, prednisolone without MTX was administered orally at a dose of $30 \mathrm{mg}(1 \mathrm{mg} / \mathrm{kg} /$ day $)$ to determine the effect of corticosteroid treatment. New sclerosed plaques appeared on her back without any improvement in contracture. She complained of general fatigue and nausea. Steroids were tapered to the minimum dose (5 mg/day), and MTX at a dose of $10 \mathrm{mg}$ weekly was started again. Under this treatment, her health improved and she has been in a relatively good condition without any side effects of MTX up to the present. MRI of her upper extremities did not display any bone abnormalities. A written informed consent was obtained from the patient and her parent.

Case 4- A nine-year-old girl presented with sclerotic plaques associated with hypopigmentation on the left lower leg. The lesions extended gradually to the entire left leg, and the sclerotic plaques were characterized by hypopigmentation and hyperpigmentation
(mLoSSI: 12, LoSDI: 8). Her laboratory findings were normal. After admission, two courses of high-dose intravenous methylprednisolone (30 $\mathrm{mg} / \mathrm{kg}$ ), each containing three pulses, were administered for two consecutive weeks (two years since patient realized symptoms). The patient was treated with a consecutive tapering of oral prednisolone $(0.5 \mathrm{mg} / \mathrm{kg})$ and maintenance treatment with oral MTX (10 $\mathrm{mg} / \mathrm{m}^{2} /$ week). The sclerotic plaques and the affected areas of her left leg improved gradually. Adverse events or relapse were not noted. The patient was effectively treated with a combination of systemic corticosteroids and MTX. MRI displayed no bone abnormalities. A written informed consent was obtained from the patient and her parent.

\section{DISCUSSION}

Localized scleroderma is a rare disease, particularly in children, and its clinical features have been categorized into the following three morphological variants: morphea, generalized morphea, and linear scleroderma. Researchers from the Mayo Clinic identified 235 cases of localized scleroderma, which included all variants, in the last 30 years. Furthermore, of these, there were only two patients younger than 10 years with generalized morphea. ${ }^{6}$ This disease may cause permanent morbidity and functional disability. ${ }^{5-7}$ Patients may have contracture, growth failure of the limbs, decreased range of motion in the joints, and limb shortening. Consequently, numerous therapeutic agents including immunomodulators have been used for treatment, although controlled studies analyzing their efficacy are rare.

There are very few detailed reports on children with localized scleroderma in Japan. Most patients with joint complications are treated with steroids. ${ }^{8}$ Early treatment would enable an improved prognosis to prevent short stature and contractures of joints, particularly in childhood. Uziel et al. ${ }^{5}$ reported that MTX and steroid therapy were effective in all children with localized scleroderma, including six patients with generalized morphea. However, they also reported that MTX treatment improved the range of motion in only two out of 10 pediatric patients with generalized morphea. ${ }^{9}$ 
In our study, MTX and corticosteroids were used to treat several types of contractures. The patients' skin sclerotic lesions improved and became smaller, and some sclerotic lesions disappeared completely (case 4). Osteomyelitis was identified on MRI, although other disease markers, including soluble IL-2 receptor levels were within the normal range. A recent study suggested that an increase in circulating serum levels of soluble IL-2 receptors correlated with disease severity in patients with morphea. ${ }^{10}$ However, two patients (cases 1 and 2) in our study showed osteomyelitis under their skin lesions, which leads to an asymmetry of the extremities. Therefore, MRI is a very useful tool to determine the depth of skin lesions and to evaluate the effectiveness of treatments.

In case 2, when the dose of corticosteroids was tapered, new sclerotic lesions and arthralgia appeared. In our patients, MTX was insufficient to resolve sclerotic skin lesions, and corticosteroids were additionally required.

Magnetic resonance imaging has been shown to be useful for the detection of musculoskeletal manifestations in patients with localized scleroderma. MRI imaging of morphea displays a low signal on T1WIs and a high signal on STIR images or enhanced $T_{1}$ imaging, owing to thickening of the dermis and infiltration of subcutaneous adipose tissue. ${ }^{11,12}$ In 1994, Liu et al. ${ }^{13}$ reported that a 14 -year-old boy with a leg length discrepancy, muscle atrophy, and bone modelling deformity displayed abnormal signals on MRI. The degree of inflammation infiltration to the collagen tissue of the subcutaneous fascia and muscle are correlated with signal intensity. When infiltration occurs in the bone, $\mathrm{T}_{2}$ and enhanced $\mathrm{T}_{1}$ images show a zonular high signal, which is the opposite of normal bone marrow, as in osteomyelitis. ${ }^{11,12}$ Schanz et al. ${ }^{11}$ reported that musculoskeletal involvement was detected in 32 out of 43 patients with localized scleroderma. However, only two cases (5\%) showed bone marrow involvement. They found that two out of the seven children younger than 18 years with bone marrow involvement identified on MRI were classified as having pansclerotic morphea. Both cases were similar to those of our study. In our present study, we detected changes on MRI in the bone marrow in two (12-year-old girl and 13-year-old girl) of the four children, without any obvious abnormalities noted by blood tests. Muroi et al. ${ }^{14}$ also reported a four-year-old girl with localized scleroderma associated with osteomyelitis, in whom MRI displayed a high signal on $\mathrm{T}_{2}$ WIs. Horger et al. ${ }^{12}$ and Liu et al. ${ }^{13}$ also described children who showed bone marrow involvement. Therefore, bone marrow involvement might be a characteristic of pediatric localized scleroderma. On the other hand, findings of dermis thickening and infiltration of subcutaneous adipose tissue, fascia, intrafascial diaphragm, synovial bursitis, and enthesitis were not obvious in the reported cases and in our four cases. Therefore, such symptoms may require a longer duration of disease than those in the reported cases. Systemic scleroderma is not associated with bone marrow involvement, although there is a high positive ratio of fasciitis and subcutaneous thickening, as reported by Schanz et al. ${ }^{15}$ Although the precise mechanism of bone marrow edema remains unclear, a direct effect of affected skin might cause edema by cytokines or other molecules.

In general, approximately $40 \%$ of patients with generalized morphea show arthralgia, preceded by contractures and disability. Consequently, systemic therapies with MTX and corticosteroids should be started at an early stage, when the sclerotic skin lesions are progressing and joint complications first appear. Controlled studies of the efficacy of these drugs in Japan are required to clarify the effects and to find an effective treatment for generalized morphea in children. To help determine treatments, MRI is highly useful because it can evaluate invasion depth. ${ }^{16}$ Thermography might be useful additionally to evaluate the expansion of sclerotic lesions, as we reported previously. ${ }^{17}$

Because of the natural healing tendencies of localized scleroderma, topical treatments are generally given. In advanced cases, oral steroids (prednisolone 20-30 $\mathrm{mg}$ as an initial amount), topical tacrolimus, oral cyclosporine, oral MTX, oral cyclophosphamide, immunosuppressant therapy, PUVA therapy (oral, topical, or both), and mycophenolate mofetil have been used. ${ }^{18,19}$ Oral steroid and immunosuppressive agents might improve their prognosis. However, early diagnosis and intervention by early treatment are required for functional improvement. Thus, we suggest that MRI is clinically useful to facilitate disease 
control and evaluate patients' responsiveness to various treatments.

\section{Declaration of conflicting interests}

The authors declared no conflicts of interest with respect to the authorship and/or publication of this article.

\section{Funding}

The authors received no financial support for the research and/or authorship of this article.

\section{REFERENCES}

1. Murray KJ, Laxer RM. Scleroderma in children and adolescents. Rheum Dis Clin North Am 2002;28:60324.

2. Peterson LS, Nelson AM, Su WP. Classification of morphea (localized scleroderma). Mayo Clin Proc 1995;70:1068-76.

3. Christen-Zaech S, Hakim MD, Afsar FS, Paller AS. Pediatric morphea (localized scleroderma): review of 136 patients. J Am Acad Dermatol 2008;59:385-96.

4. Beltramelli M, Vercellesi P, Frasin A, Gelmetti C, Corona F. Localized severe scleroderma: a retrospective study of 26 pediatric patients. Pediatr Dermatol 2010;27:476-80.

5. Uziel Y, Krafchik BR, Silverman ED, Thorner PS, Laxer RM. Localized scleroderma in childhood: a report of 30 cases. Semin Arthritis Rheum 1994;23:328-40.

6. Christianson HB, Dorsey CS, Kierland RR, O'leary PA. Localized scleroderma; a clinical study of two hundred thirty-five cases. AMA Arch Derm 1956;74:629-39.

7. Diaz-Perez JL, Connolly SM, Winkelmann RK. Disabling pansclerotic morphea of children. Arch Dermatol 1980;116:169-73.

8. Kawashima H, Watanabe C, Kashiwagi Y, Sato S, Ioi H, Sasamoto M, et al. Therapy of childhood generalized morphea: case reports and reviews of the literature of Japanese cases. Pediatr Int 2006;48:342-5.
9. Uziel Y, Feldman BM, Krafchik BR, Yeung RS, Laxer RM. Methotrexate and corticosteroid therapy for pediatric localized scleroderma. J Pediatr 2000;136:91-5.

10. Uziel Y, Krafchik BR, Feldman B, Silverman ED, Rubin LA, Laxer RM. Serum levels of soluble interleukin-2 receptor. A marker of disease activity in localized scleroderma. Arthritis Rheum 1994;37:898-901.

11. Schanz S, Fierlbeck G, Ulmer A, Schmalzing M, Kümmerle-Deschner J, Claussen CD, et al. Localized scleroderma: MR findings and clinical features. Radiology 2011;260:817-24.

12. Horger M, Fierlbeck G, Kuemmerle-Deschner J, Tzaribachev N, Wehrmann M, Claussen CD, et al. MRI findings in deep and generalized morphea (localized scleroderma). AJR Am J Roentgenol 2008;190:32-9.

13. Liu P, Uziel Y, Chuang S, Silverman E, Krafchik B, Laxer R. Localized scleroderma: imaging features. Pediatr Radiol 1994;24:207-9.

14. Muroi E, Ogawa F, Yamaoka T, Sueyoshi F, Sato $\mathrm{S}$. Case of localized scleroderma associated with osteomyelitis. J Dermatol 2010;37:81-4.

15. Schanz S, Henes J, Ulmer A, Kötter I, Fierlbeck G, Claussen $C D$, et al. Magnetic resonance imaging findings in patients with systemic scleroderma and musculoskeletal symptoms. Eur Radiol 2013;23:212-21.

16. Schanz S, Henes J, Ulmer A, Kötter I, Fierlbeck $G$, Claussen CD, et al. Response evaluation of musculoskeletal involvement in patients with deep morphea treated with methotrexate and prednisolone: a combined MRI and clinical approach. AJR Am J Roentgenol 2013;200:376-82.

17. Kashiwagi Y, Kawashima H, Agata K, Akaba M, Hirose A, Go S, et al. Thermography for evaluation of localized scleroderma treated with methotrexate and corticosteroid. Indian J Pediatr 2013;80:980-1.

18. Torok KS. Pediatric scleroderma: systemic or localized forms. Pediatr Clin North Am 2012;59:381-405.

19. Zulian F, Vallongo C, Patrizi A, Belloni-Fortina A, Cutrone $\mathrm{M}$, Alessio $\mathrm{M}$, et al. A long-term follow-up study of methotrexate in juvenile localized scleroderma (morphea). J Am Acad Dermatol 2012;67:1151-6. 